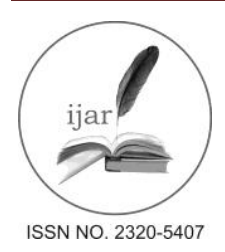

Journal homepage: http://www.journalijar.com
Journal DOI: 10.21474/IJAR01

INTERNATIONAL JOURNAL

RESEARCH ARTICLE

\title{
CLINICAL RELEVANCE OF INTERFERON-GAMMA RELEASE ASSAY VERSUS TUBERCULIN SKIN TESTING FOR DIAGNOSIS OF LATENT TUBERCULOSIS IN CHRONIC HEMODIALYSIS PATIENTS.
}

\author{
Hussien Ahmed Abu Elyazeid ${ }^{1}$, Ghanem Abdellatef Mohamed ${ }^{1}$, Khaled Mostafa ${ }^{1}$, \\ Hydi Ahmed ${ }^{2}$, Ahmed Aly ${ }^{3}$. \\ 1. Departments of Chest Diseases, Faculty of Medicine, Al Azhar University,Egypt. \\ 2. Departments Clinical Pathology,Faculty of Medicine, Sohag University, Egypt. \\ 3. Internal Medicine, Faculty of Medicine, Sohag University, Egypt.
}

\section{Manuscript Info}

Manuscript History:

Received: 12 May 2016

Final Accepted: 19 June 2016

Published Online: July 2016

Key words:

Tuberculosis, Quantiferon-TB Gold,

Tuberculin skin test, End stage renal disease.

\section{*Corresponding Author}

\section{Hydi Ahmed,}

Department of Clinical Pathology,

Sohag University, Egypt E-mail:

hydi.ahmed@yahoo.com

\begin{abstract}
There is no gold standard method for detecting latent tuberculosis infection (LTBI) in end stage renal disease (ESRD), the use of tuberculin skin test(TST) remains controversial due to its high rate of false results, new interferon gamma release assays have been developed for diagnosing LTBI as the QuantiFERON-TB Gold test(QFT-G).

The aim of this work:- was to evaluate the value of Quantiferon-TB Gold test and compare its performance with tuberculin skin test in the diagnosis of latent tuberculosis infection among ESRD patients receiving hemodialysis(HD).

Patients and Methods:- 60 ESRD patients undergoing HD and 40 healthy controls were subjected to TST and QFT-G to diagnose Latent TB infection.

Results:- QFT-G and TST were positive in $31.7 \%$ and $45 \%$ of patients respectively. The overall agreement was $73.3 \%$ (statistically significant with moderate agreement, $\mathrm{p}=0.001 \& \mathrm{k}=0.44$ ), while it was $70 \%$ in $\mathrm{BCG}$ vaccinated patients (statistically significant with fair agreement, $\mathrm{p}=0.003$ \& $\mathrm{k}=0.37$ ) and $80 \%$ in non BCG vaccinated patients (statistically significant with moderate agreement, $\mathrm{p}=0.001 \& \mathrm{k}=0.56$ ). There were no statistically significant differences between patients and controls as regards age, sex, duration of hemodialysis and history of contact to known TB patients.

Conclusion:- We conclude that QFT-G test shows overall moderate agreement with TST and it could be used in screening of LTBI in ESRD patients simultaneously with TST and QFT-G test is more specific and accurate than TST in BCG vaccinated patients as BCG vaccination reduces their agreement, increasing the risk of TST false positive results rather than QFT-G positivity but relatively high cost and need for laboratory instruments is a limitation of QFT-G test
\end{abstract}

\section{Introduction:-}

Mycobacterium tuberculosis infection is a major global public health problem. The detection and monitoring of $M$. tuberculosis infections are essential to control its spread. Tuberculosis (TB) remains one of the major causes of death from a single infectious agent worldwide. Since there is no cure for some multidrug-resistant strains of $M$. tuberculosis, there is concern that they may spread around the world, stressing the need for additional control measures, such as new diagnostics, better drugs for treatment and a more effective vaccine (WHO 2004). Contemporary estimates argue that one- third of the earth's population is already infected (WHO 2009). Egypt is ranked among the mid-level incidence countries (National TB control Program 2008). About $90 \%$ of those infected with Mycobacterium tuberculosis are asymptomatic, sometimes called latent TB infection (LTBI), with only a 10\% lifetime chance that a latent infection will progress to active TB (CDC 2005). 
The tuberculin skin test (TST) predominates as the method of choice for detection of latent TB infections (LTBI). Although widely used, it cannot be trusted as a "gold standard" because of the variability of interpretation and false positives and negatives. Placement of the purified protein derivative (PPD), subjective reading of the results, and the unwillingness of individuals to return for test interpretation are responsible for many problems associated with its use. In addition, environmental mycobacteria and the Mycobacterium bovis Bacillus Calmette-Guérin (BCG) vaccine commonly cause false-positive results (Taggart et al, 2004).

End stage renal disease (ESRD) patients are at increased risk of latent tuberculosis infection (LTBI) compared to the general population. LTBI is more likely to progress to TB infection in ESRD. Investigations from several countries have shown that the increased risk of TB among patients on long-term hemodialysis is 6.9 to 52.5 times higher than the rate in the general population (Hussein et al; 2002).

Although guidelines recommend screening these patients for LTBI, the tuberculin skin test is believed to be insensitive in ESRD patients and might be due to the immune-compromised condition (Hayriye et al; 2011). In addition, the TST might be falsely positive in persons with a history of previous non tuberculous mycobacterium (NTM) infection or vaccination with Bacillus Calmette-Guérin (BCG) (Wang etal; 2002).

Immune response to mycobacterial infections is primarily a cell-mediated immune response to $M$. tuberculosis antigens. When the $\mathrm{T}$ cells are stimulated by purified protein derivative from $M$. tuberculosis, they produce the cytokine gamma interferon (IFN- $\gamma$ ), which allows macrophages to kill the intracellular pathogen (Andersen etal; 2000). Researches have succeeded in the development of two commercial IFN- $\gamma$ assays, the QuantiFERON-TB assay (Cellestis Limited, Carnegie, Victoria, Australia) and the T SPOT-TB assay (Oxford Immunotec, Oxford, UK). The Quantiferon-TB assay detects the in vitro cell-mediated immune response to M. tuberculosis infection by measuring IFN- $\gamma$ in whole blood that was incubated with $M$ tuberculosis antigens (early secreted antigenic target 6 (ESAT6) and culture filtrate protein 10 (CFP10)) (WHO 2009),. The T SPOT-TB assay, uses ESAT6 and CFP10, and detects the number of IFN- $\gamma$ producing $\mathrm{T}$ cells in peripheral blood mononuclear cells (PBMCs) based on ELISPOT (Andersen, 2000), The use of QuantiFERON-TB Gold (QFT-G) for detection of LTBI appears to be more promising than tuberculin skin test in hemodialysis patients( Hayriye etal; 2011).

The aim of this study was to evaluate the value of Quantiferon-TB Gold test and compare its performance with tuberculin skin test in the diagnosis of latent tuberculosis infection among ESRD patients receiving hemodialysis.

\section{Patients and methods:-}

This study was carried out on 60 patients (33 males and 27 females with age ranging from 39 to 70 years) with chronic renal failure undergoing hemodialysis, from the dialysis unit of the Internal Medicine department, Sohag University, Egypt during the period from March 2014 to March 2015. In addition, 40 apparently healthy persons (24 males and 16 females with age ranging from 40 to 71 years) were recruited as a control group. The study protocol was approved by the local ethics committee and all patients gave their consent prior to the study.

\section{Inclusion criteria:-}

Enrolled patients were (1) Chronic renal failure patients undergoing hemodialysis for $>6$ months (2) Patients with no symptoms or signs of tuberculosis. (3) Sputum smear negative for acid fast bacilli.

\section{Exclusion criteria:-}

(1) Patients with active TB (2) Chest $X$ ray findings of pulmonary TB (3) Co-morbidity leading to immune suppression such as diabetes mellitus, liver disease, under corticosteroid treatment.

Both patients and controls groups were subjected to the followings:

- History taking including: age, sex, clinical diagnosis, type of treatment, co morbidity, history of BCG vaccination and history of contact to TB patient.

- Clinical assessment: Chest X-ray, complete blood count, renal function tests and Ziehl Neelsen stained sputum smear for acid fast bacilli.

Tuberculin Skin Test (TST) (Mantoux test):-

TST was performed by intradermal injection of $0.1 \mathrm{ml}$ of PPD tuberculin ( 5 units) into the skin of the ventral aspect of forearm, performing a wheal 6 to $10 \mathrm{~mm}$ in diameter (Figure 1a),. The test was then read 48 to 72 hours later. 
The presence of induration was determined by touch, Erythema was not considered and the induration diameter was measured in millimeters (Figure $\mathbf{1 b}$ ), $10 \mathrm{~mm}$ or more was considered as positive results (Huebner et al., 1993, CDC 2005)

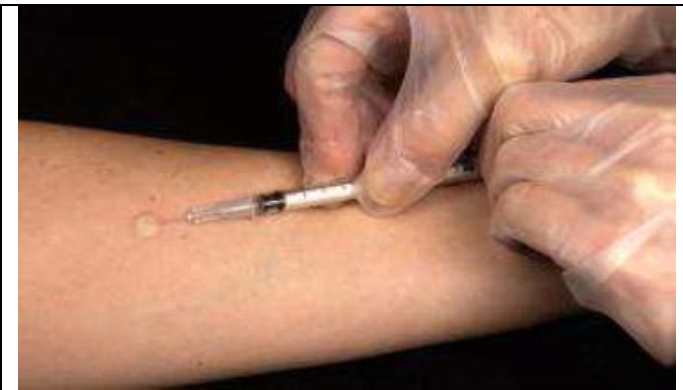

Figure (1a):- Tuberculin Skin Test

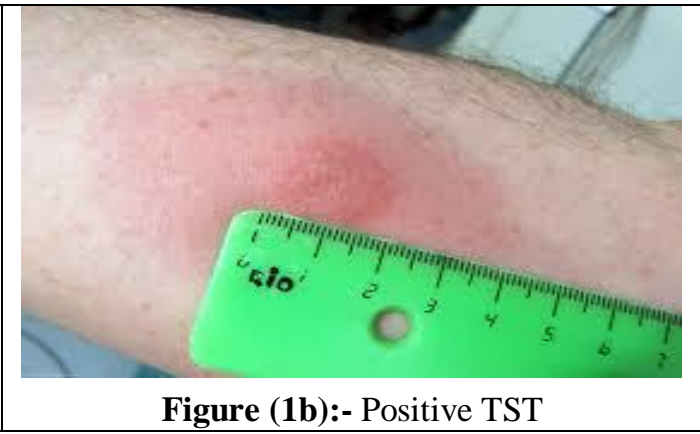

Figure (1b):- Positive TST

\section{QuantiFERON -TB Gold test(QFT-G test):-}

QFT-G test is an interferon- $\gamma$ release assay (IGRA) that uses two specialized blood collection tubes that contain certain M. tuberculosis proteins (ESAT-6, CFP-10) and negative (Nil) control tubes (CDC 2006).

The test was performed as described by manufacturer (Cellestis Limited, Carnegie, Victoria, Australia): $1 \mathrm{ml}$ of blood was taken by venipuncture directly into each of the collection tubes, shaking the tubes vigorously for 5 seconds (10 times) was done to ensure that the entire inner surface of the tube has been coated with blood. The tubes were then transferred to a $37^{\circ} \mathrm{C}$ incubator for $16-24$ hours, after which plasma was harvested and tested for the presence of IFN- $\gamma$ produced in response to the peptide antigen using the quantitative sandwich enzyme immunoassay technique (ELISA) by the Stat fax 2600 microplate reader (Awareness Technologies, Palm City, USA ). Positive test was considered if the IFN- $\gamma$ response to TB antigens was above the test cut off $(\geq 0.35 \mathrm{IU} / \mathrm{ml})$ after subtracting the background IFN- $\gamma$ response in the negative control (Gerogianni, et al., 2008).

\section{Statistical methods:-}

Statistical Package for Social Science (SPSS) program version 16 was used for analysis of data. Data were presented in number (percent) or median and interquartile range, Chi-square (x2) test was used for qualitative data, Significant correlation between different variables was determined using Pearson's correlation coefficient, The agreement between TST and QFT-G test results was assessed using kappa (k) coefficient (poor agreement: $<0.2$, fair: 0.2 to 0.4 , moderate: 0.4 to 0.6 , good: 0.6 to 0.8 , very good: 0.8 to 1.0 ), significant results are considered when a probability value $(\mathrm{P}$ value) $<0.05$, highly significant $\mathrm{P}<0.01$, very highly significant $\mathrm{P}<0.001$, non-significant $\mathrm{P}>$ 0.05

\section{Results:-}

The study included 60 chronic renal failure patients (33 males and 27 females), age range from 39 to 70 years with hemodialysis duration $36 \pm 15$ months and 40 healthy control subjects ( 24 males and 16 females), the age range of the controls was 40 to 71 years. History of BCG vaccinations was present in $40(66.7 \%)$ patients and 28(70\%) controls; also a history of contact to T.B patient was present in $9(15 \%)$ patients and $4(10 \%)$ controls. There were no significant statistical differences found between patients and controls regarding the previous demographic data (P>0.05) (Table 1)

Table 1:- Demographic data of the study groups:-

\begin{tabular}{|l|c|c|}
\hline \multicolumn{1}{|c|}{ Demographic data } & Patients group (n=60) & Controls group(n=40) \\
\hline Sex : $\quad$ Male & $33(55 \%)$ & $24(60 \%)$ \\
& $27(45 \%)$ & $16(40 \%)$ \\
\hline Age (years) & $55.1(39-70)$ & $56.2(40-71)$ \\
\hline History of BCG vaccinations & $40(66.7 \%)$ & $28(70 \%)$ \\
\hline History of contact to T.B patient & $9(15 \%)$ & $4(10 \%)$ \\
\hline Duration of hemodialysis (months) & $36 \pm 15$ & 0 \\
\hline
\end{tabular}

Data were expressed as number (percentage), or mean (range). 


\section{Results of Tuberculin Skin Test (TST) and Quantiferon-TB Gold Test (QFN-G):-}

Positive TST results (induration cut off $10 \mathrm{~mm}$ ) were found in 27/60 (45\%) and 4/40 (10\%) of hemodialysis patients and controls respectively, while positive QFT-G test results were found in 19/60 (31.7\%) and 1/40 (2.5\%) of hemodialysis patients and controls respectively (Table 2).

Table 2:- Comparison between TST and QFT-G results in the study groups.

\begin{tabular}{|c|c|c|c|}
\hline Results & Patient group (n= 60) & Control group(n= 40) & P value \\
\hline \multicolumn{2}{|c|}{ Tuberculin Skin Test (TST) } & $<0.001$ \\
\hline Positive & $27(45 \%)$ & $4(10 \%)$ & $<0.001$ \\
\hline Negative & $33(55 \%$ & $36(90 \%)$ & $<0.001$ \\
\hline \multicolumn{4}{|c|}{ Quantiferon-TB Gold Test (QFT-G) } \\
\hline Positive & $19(31.7 \%)$ & $1(2.5 \%)$ & $<0.01$ \\
\hline Negative & $41(68.3 \%)$ & $39(97.5 \%)$ & \\
\hline
\end{tabular}

* Data were expressed as number (percentage),

\section{Agreement of TST and QFT-G for detecting LTBI:-}

In the patients group we found that 15 cases were positive in both TST and QFT-G, 12 cases were positive in TST and negative in QFT-G, 4 cases were positive in QFT-G and negative in TST and 29 Cases were negative in both tests, so the overall agreement between QFT-G and TST in patients was 44/60 (73.3\%), this was found to be statistically significant with moderate agreement $(\mathrm{p}=0.001 \& \mathrm{k}=0.44)$ (Table 3 ), however in the control group we found that only 1 of the controls was positive and 36 were negative in both tests with poor agreement ( $\mathrm{p}=0.5 \&$ $\mathrm{k}=0.16$ ) (Table 4).

Table 3:- Agreement of TST and QFT-G in patient group.

\begin{tabular}{|l|c|c|c|c|}
\hline \multirow{2}{*}{ Test } & \multicolumn{3}{|c|}{ TST } \\
\cline { 3 - 5 } QFT-G & Positive & Positive & Negative & Total \\
\cline { 2 - 5 } & Negative & $15(25 \%)$ & $4(6.7 \%)$ & $19(31.7 \%)$ \\
\cline { 2 - 5 } & Total & $12(20 \%)$ & $29(48.3 \%)$ & $41(68.3 \%)$ \\
\cline { 2 - 4 } & Agreement $(15+29) / 60=73.3 \%(\kappa=0.44 \& \mathrm{p}=0.001)$ & $33(55 \%)$ & $60(100 \%)$ \\
\hline
\end{tabular}

Table 4:- Agreement of TST and QFT-G in control group.

\begin{tabular}{|l|c|c|c|c|}
\hline \multirow{2}{*}{ Test } & \multicolumn{3}{c|}{ TST } \\
\cline { 3 - 5 } & Positive & Negative & Total \\
\hline \multirow{3}{*}{ QFT-G } & Positive & $1(2.5 \%)$ & $0(0 \%)$ & $1(2.5 \%)$ \\
\cline { 2 - 5 } & Negative & $3(7.5 \%)$ & $36(90 \%)$ & $39(97.5 \%)$ \\
\cline { 2 - 5 } & Total & $4(10 \%)$ & $36(90 \%)$ & $40(100 \%)$ \\
\hline \multicolumn{2}{|c|}{ Agreement $(1+36) / 40=92.5 \%(\kappa=0.16 \& p=0.5)$} \\
\hline
\end{tabular}

As regards BCG vaccinated persons the agreement between the two tests was fair $(70 \%, \mathrm{k}=0.37 \& \mathrm{p}=0.003)$ and poor $(97 \%, \mathrm{k}=0.0 \& \mathrm{p}=0.53)$ in patients (Table 5) and controls respectively but in non BCG vaccinated persons, the agreement between the two tests was moderate $(80 \%, \mathrm{k}=0.56 \& \mathrm{p}=0.001)$ and fair $(83 \%, \mathrm{k}=0.41 \& \mathrm{p}=0.001)$ in patients (Table 6) and controls respectively.

Table 5:- Agreement of TST and QFT-G in BCG vaccinated patients

\begin{tabular}{|c|c|c|c|c|}
\hline \multicolumn{2}{|c|}{ Test } & \multicolumn{3}{c|}{ TST } \\
\cline { 3 - 5 } QFT-G & Positive & Positive & Negative & Total \\
\cline { 2 - 5 } & Negative & $10(25 \%)$ & $3(7.5 \%)$ & $13(32.5 \%)$ \\
\cline { 2 - 5 } & Total & $19(22.5 \%)$ & $18(45 \%)$ & $27(67.5 \%)$ \\
\hline \multicolumn{4}{|c|}{ Agreement $(10+18) / 40=70 \%(\kappa=0.37 \& \mathrm{p}=0.003)$} \\
\hline
\end{tabular}


Table 6:- Agreement of TST and QFT-G in non BCG vaccinated patients

\begin{tabular}{|c|c|c|c|c|}
\hline \multirow{2}{*}{\multicolumn{2}{|c|}{ Test }} & \multicolumn{3}{|c|}{ TST } \\
\hline & & Positive & Negative & Total \\
\hline \multirow[t]{3}{*}{ QFT-G } & Positive & $5(25 \%)$ & $1(5 \%)$ & $6(30 \%)$ \\
\hline & Negative & $3(15 \%)$ & $11(55 \%)$ & $14(70 \%)$ \\
\hline & Total & $8(40 \%)$ & $12(60 \%)$ & $20(100 \%)$ \\
\hline
\end{tabular}

Regarding the history of contact to known TB cases in the current study, also positive TST and QFT-G results were found to be $90 \%(8 / 9)$ and $100 \%(9 / 9)$ respectively in hemodialysis patients with history of contact to known TB cases, however positive TST and QFT-G results were found to be 75\%(3/4) and $25 \%(1 / 4)$ respectively in controls with history of contact to known TB cases with no statistically significant differences $(\mathrm{P}>0.05)$

\section{Discussion:-}

TB elimination strategies rely on proper detection and treatment of individuals with latent tuberculosis infection (LTBI) and thereby preventing progression to active disease (Gomez etal., 2004). Although the tuberculin skin test has aided in the diagnosis of LTBI, its interpretation is difficult because sensitization with non-tuberculous Mycobacteria or previous BCG vaccination leads to false-positive tests (Garg et al., 2003). A major breakthrough in recent years has been the development of in vitro assays that measure T-cell release of IFN- $\gamma$ in response to stimulation with antigens specific to M. tuberculosis such as ESAT6 and CFP10. On the basis of this technology, a commercial IFN- $\gamma$ test is available: QuantiFERON-TB Gold assay (QFT-G) (Dominguez et al., 2008).

There are few studies evaluating the value of QFT-G test in LTBI in chronic renal failure patients receiving hemodialysis and also investigating the agreement between QFT-G test and TST(Emin et al; 2011), However, few studies have compared both tests in immunocompromised patients (Kobashi, et al., 2007; Luetkemeyer et al., 2007; Pratt et al., 2007).

The sensitivity of the TST is low in HD patient because of the cross reactivity of TST with the BCG vaccine thus, Moreover, TST results can be false negative because of biological problems, or technical problems related to the TST administration, TST material or measuring the reaction. In addition, ESRD is known to be a risk factor for false TST negativity. Sensitivity in ESRD patients declines in parallel with decreasing cellular immune system function. Due to these reasons TST is not a good marker for diagnosis in HD patients (Hayriye et al; 2011).

In the current study, positive TST results were found in $45 \%$ and $10 \%$ of hemodialysis patients and controls respectively, while positive QFT-G test results were found in $31.7 \%$ and $2.5 \%$ of hemodialysis patients and controls respectively, lower rate of positivity in the QFT-G can be explained by a higher specificity of QFT than TST.

Our results are in accordance with Emin et al. who reported in their study that the positivity of QFT-G was 39.6\%, however TST positivity differed according to induration cut off value(Emin et al; 2011)

On the other hand, Inoue et al. reported that the sensitivity and specificity of the QFT-G in hemodialysis patients was $100 \%$ and $89.7 \%$ respectively (Inoue et al. 2009) and a cross-sectional comparison study on 275 ESRD Turkish patients performed by Ates et al. revealed $46.7 \%$ of patients were tested positive by the QFT-GIT and 35.5\% were found to be TST positive (Ates et al. 2009).

In the present study the overall agreement between QFT-G and TST in hemodialysis patients was $73.3 \%$ and it was found to be statistically significant with moderate agreement, however poor agreement was found between QFT-G and the TST in controls, These results were found to be in accordance with many researchers as Hayriye and coworkers who found moderate agreement between the TST and QFT-G assay results when TST induration is above $10 \mathrm{~mm}$ (Hayriye et al; 2011)., Emin et al. who found fair to moderate agreements between QFT-G test and TST in either a TST cut off value of $5 \mathrm{~mm}$ and $10 \mathrm{~mm}$ in patients with renal failure receiving $\mathrm{HD}$ (Emin et al; 2011), and Ates et al. who found poor concurrence (Ates et al. 2009).

In an Egyptian study performed comparing QFT-G and TST in diagnosis LTBI among chronic renal failure patients, Abdel-Nabi et al found that although both tests gave comparable results, yet there was a discrepancy between both. $\mathrm{TST}+/ \mathrm{QFT}+$ group was $10 \%$, TST $+/ \mathrm{QFT}-$ group was $5 \%$, TST $-/ \mathrm{QFT}+$ was $10 \%$ and $\mathrm{TST}-/ \mathrm{QFT}-$ group was 
75\%.(Abdel-Nabi etal., 2014), , also another Egyptian study performed by Mohammad et al to screen LTBI in 50 cancer patients using 2-step tuberculin skin test and QFT-GIT test revealed that QFT-GIT test was more sensitive in detecting M. tuberculosis infection in patients with malignancy than the TST, a poor agreement was found between QFT-GIT and TST-I results and a moderate agreement was found between QFT-GIT and TST-II results (Mohammad etal., 2015) and Abdel Samea etal found good agreement between tuberculin test and QFT-Gold IT test where the ' $\kappa$ ' was 0.65 (Abdel Samea etal 2013).

A study reported by Diel et al. showed that QFT-G is a more accurate indicator of the presence of LTBI than the TST and provides at least the same sensitivity for detecting individuals who will progress to active TB. Also, high sensitivity and specificity of QFT-G tests in active tuberculosis disease is reported (Diel et al; 2011)

In a recent study from Turkey, Seyhan et al. reported an overall agreement of 65\% between the QFT-G and the TST at a cut off value of $10 \mathrm{~mm}$ (Seyhan et al., 2010), also Lee et al. reported the overall agreement between QFT-G and TST as fair $(\mathrm{k}=0.34)$ when the TST cut off was defined as $5 \mathrm{~mm}$ and fair $(\mathrm{k}=0.25)$ when it was defined as $10 \mathrm{~mm}$. They concluded that the QFT-G was a more accurate method for identifying those truly infected with Mycobacterium tuberculosis, even in BCG-vaccinated individuals (Lee et al. 2009). Also, the agreement between TST and QFT-G was poor $(\mathrm{k}=0.16, \mathrm{p}=0.116)$ by Triverio et al who defined TST positive if an induration diameter was $>5 \mathrm{~mm}$ and found that QFT-G was twice as effective at detecting probable LTBI (46\%) as TST (25\%) (Triverio etal; 2009). Another study by Winthrop et al., tested 100 patients with ESRD who received hemodialysis and they reported that QFT-G and T-SPOT-TB might offer a better method for detecting TB infection in ESRD patients (Winthrop et al., 2008)

In our study the rate of BCG vaccination was $66.7 \%$ and $70 \%$ for patients and controls respectively, similar results were reported by Emin et al, However previous studies revealed a BCG vaccination rate between $77.6 \%$ and $90 \%$ (Ozdemir et al., 2007, Soysal et al., 2008, Elbek et al., 2009, Emin et al; 2011).

In the present study the overall agreement between the QFT-G and the TST in BCG non vaccinated hemodialysis patients was $80 \%$ and it was found to be statistically significant with moderate agreement $(\mathrm{k}=0.56 \& \mathrm{p}=0.001)$, however fair agreement was found between QFT-G and the TST in controls. As regard to BCG vaccination the agreement between the two tests was fair $(70 \%, \mathrm{k}=0.37 \& \mathrm{p}=0.003)$ and poor $(97 \%, \mathrm{k}=0.0 \& \mathrm{p}=0.53)$ in patients and controls respectively because BCG vaccination reduced the agreement, influencing the TST positivity (rather than QFT-G positivity) and increasing the risk of TST false positive results.

These results were found to be in accordance to Emin et al who found in their study on 64 BCG vaccinated cases with renal failure receiving HD that the agreements between QFT-G test and TST in either a TST cut off value of 5 $\mathrm{mm}$ and $10 \mathrm{~mm}$ to be moderate and fair $(k=0.432 \& k=0.229$ respectively). In the non-vaccinated 29 cases, the overall agreement between the two tests were both fair $(\mathrm{k}=0.322, \& \mathrm{k}=0.228$, respectively) for TST cut off value of 5 $\mathrm{mm}$ and $10 \mathrm{~mm}$ respectively (Emin et al; 2011)

Hayriye and coworkers reported a history of BCG vaccination in $80 \%$ of cases, however no statistically significant difference was detected in the TST and QFT-G test results between vaccinated and unvaccinated patients (P value $=0.221 ; 0.454$ respectively). This might be explained by the fact that, in their study group, only 2 cases were positive by QFT-G: one of them was BCG vaccinated and the other one was not (Hayriye et al; 2011)., In addition, Franken et al. found no significant relation between BCG vaccinated patients and TST and QFT-G test results (Franken et al 2007), however Abdel-Samea et al. revealed that QFT-Gold IT, has excellent sensitivity and specificity unaffected by BCG vaccination and tuberculin test specificity is high in non-BCG-vaccinated populations but low and variable in BCG vaccinated populations (Abdel samea et al., 2013).

A meta-analysis study done by Pai and O'Brien (2008) revealed that the pooled sensitivity was 78\% QuantiFERONTB Gold, and 70\% for QuantiFERON-TB Gold In-Tube. The pooled specificity for both QuantiFERON tests was 99\% among non-BCG-vaccinated participants and 96\% among BCG-vaccinated participants. They concluded that the interferon gamma release assays (IGRAs), especially QuantiFERON-TB Gold and QuantiFERON-TB Gold InTube, have excellent specificity that is unaffected by BCG vaccination (Pai and O'Brien 2008).

In the current study positive TST and QFT-G results were found to be $90 \%$ and $100 \%$ respectively in hemodialysis patients with history of contact to known TB cases, but Ates et al. revealed that QFT-GIT and TST results were not 
associated with contact to tuberculosis (Ates etal., 2009), however Connell et al. compared the performance of TST and QFT-G test in a total of 75 subjects who were contacts of active tuberculous patients and they found that concordant results between the two tests existed as patients with positive TST had positive QFT-G test and patients with negative TST had negative QFT-G (Connell et al. 2011).

As regards age, sex and duration of hemodialysis, there was no statistically significant relation between results of TST or QFT-G in the present study, similarly, Emin et al found no significant relation between the two tests regarding age and sex (Emin et al; 2011), on the other hand, our results disagree with Tegbaru et al. who found more positive results in males than females. This difference may be attributed to the limited number of patients in our study group (60 patients) compared to their study which was performed on 1286 individuals (Tegbaru et al., 2006).

In conclusion, the lower rate of QFT-G test positivity in screening of LTBI in patients with ESRD can be explained by its higher specificity than TST, QFT-G test shows overall moderate agreement with TST and it could be used simultaneously with TST and it is more specific and accurate than TST in BCG vaccinated patients as BCG vaccination reduces their agreement, influencing the TST positivity rather than QFT-G positivity and increasing the risk of TST false positive results, but relatively high cost and need for laboratory instruments is a limitation of QFT$\mathrm{G}$ test and to analyze the use of it alone in screening of LTBI, large longitudinal studies are required.

\section{References:-}

1. Abdel-Nabi E.A, Eissa S.A., Soliman Y.M.A., Amin W.A. (2014): Quantiferon vs. tuberculin testing in detection of latent tuberculous infection among chronic renal failure patients. Egyptian Journal of Chest Diseases and Tuberculosis Volume 63, Issue 1, January 2014, Pages 161-165.

2. Abdel-Samea S.A, Ismail Y.M, Fayed S.M.A, Mohammad A.A (2013): Comparative study between using QuantiFERON and tuberculin skin test in diagnosis of Mycobacterium tuberculosis infection. Egyptian Journal of Chest Diseases and Tuberculosis. Volume 62, Issue 1, January 2013, Pages 137-143

3. Andersen P, Munk ME, Pollock JM and Doherty TM. (2000): Specific immune based diagnosis of tuberculosis. Lancet 356: 1099-1104.

4. Ates. G, Ozekinci.t, Yildiz.T, Danis. R (2009): Comparison of interferon gamma release assay versus tuberculin skin test for latent tuberculosis screening in hemodialysis patients. Biotechnology \& Biotechnological Equipment ISSN: $1310-2818$ 1314-3530

5. Centers for Disease Control and Prevention (2005): National plan for reliable tuberculosis laboratory services sing a systems approach: recommendations from CDC and the Association of Public Health Laboratories Task Force on Tuberculosis Laboratory Services. MMWR;54(No. RR-6).

6. Centers for Disease Control and Prevention (2006): Emergence of Mycobacterium tuberculosis with extensive resistance to second-line drugs - worldwide. MMWR 55 (11): 301-305.

7. Connell DW, Singanayagam A, Charif R, George PM, Molyneaux PL, McCrudden C, Magtoto M, Harden E, Seneviratne SL, Duncan ND, Kon OM (2011): A comparison between interferon gamma release assays and the tuberculin skin test in the contact tracing of patients with chronic kidney disease. Thorax, 66:729-730.

8. Diel R, Loddenkemper R, Niemann S, Meywald-Walter K, Nienhaus A. (2011): Negative and positive predictive value of a whole-blood interferon- $\gamma$ release assay for developing active tuberculosis: an update. Am J Respir Crit Care Med. Jan 1; 183(1):88-95.

9. Dominguez J, Manzano J, Galvao MDS, Latorre I, Milà C, Blanco S, Jiménez A, Prat C, Lacoma A, Altet N and Vicente Ausina1 V. (2008): Comparison of Two Commercially Available Gamma Interferon Blood Tests for Immunodiagnosis of Tuberculosis. Clin. Vaccine Immunol. 15(1): 45-52.

10. Elbek O, Uyar M, Aydin N, Börekçi S, Bayram N, Bayram H, Dikensoy O (2009): Increased risk of tuberculosis in patients treated with antitumor necrosis factor alpha. ClinRheumatol.;28(4):421-6.

11. Emin Maden, T. TahaBekci, RecepKesli, HuseyinAtalay, TurgutTeke, YalcinSolak, Suleyman Turk, KursatUzun and RamazanKoylu (2011): Evaluation of performance of quantiferon assay and tuberculin skin test in end stage renal disease patients receiving hemodialysis. New Microbiologica, 34, 109-356.

12. Franken WP, Timmermans JF, Prins C, Slootman EJ, Dreverman J, Bruins H, van Dissel JT and Arend SM. (2007):Comparison of Mantoux and QuantiFERON TB Gold tests for diagnosis of latent tuberculosis infection in Army personnel. Clin Vaccine Immunol. Apr;14(4):477-480.

13. Garg SK, Tiwari RP, Tiwari D, Singh R, Malhotra D, Ramnani VK, Prasad GB, Chandra R and Fraziano M. (2003): Diagnosis of tuberculosis: available technologies, limitations and possibilities. J. Clin. Lab. Anal. 17:155-63.

14. Gerogianni I, Papala M, Klapsa D, Zinzaras E, Petinaki E and Gourgoulianis KI.. (2008): Whole-blood interferon-y assay for the diagnosis of tuberculosis infection in an unselected Greek population. Respirology. 13: 270274. 
15. Gomez, J.E., McKinney J.D, Muñoz-Elías EJ, Timm J, Botha T and Chan WT. (2004):M. tuberculosis persistence, latency and drug tolerance. Tuberculosis (Edinburgh, Scotland); 84(1-2): 29-44.

16. Hayriye Sayarlioglu, Mustafa Gol, Canan Eren Dagli, Ekrem Dogan, Murat Sahini, Mehmet Ali Uar, Nurhan Koksal, Mehmet Sayarlioglu and MumtazKerim Tahta(2011): Quantiferon-TB Gold test for screening latent tuberculosis infection in hemodialysis patients. Thoracic tuberculosis magazine, ;59(2): 105-110

17. Huebner RE, Schein MF and Bass JB(1993):The tuberculin skin test Review. Jr. Clin Infect Dis. Dec;17(6):968-75.

18. Hussein MM, Mooij MJ and Roujouleh H (2002): Tuberculosis and chronic renal disease. Semin Dial; 16: 38-44.

19. Inoue T, Nakamura T, Katsuma A, Masumoto S, Minami E and Ka- tagiriD. (2009): The value of QuantiFERON TB-Gold in the diagnosis of tuberculosis among dialysis patients. Nephrol Dial Transplant; 24: 2252-7.

20. Kobashi Y, Mouri K, Obase Y, Fukuda M, Miyashita N and Oka M. (2007): Clinical evaluation of QuantiFERON TB-2G test for immune compromised patients. EurRespir J 30: 945-50.

21. Lee SS, Chou KJ, Su IJ, Chen YS, Fang HC, Huang TS, Tsai HC, Wann SR, Lin HH and Liu YC. (2009): High prevalence of latent tuberculosis infection in patients in end-stage renal disease on hemodialysis: comparison of QuantiFERON-TB GOLD, ELISPOT, and tuberculin skin test. Infection; 37: 96-102.

22. Luetkemeyer A.F., Charlebois E.D., Flores L.L., Bangsberg D.R., Deeks S.G., Martin J.N. (2007): Comparison of an interferon-gamma release assay with tuberculin skin testing in HIV-infected individuals. Am. J. Respir. Crit. Care Med. 175, 737-742.

23. Mohammad H.A, Esmail M.A, Abdelftah M.T, Soliman W, mohammad A, Esmail E (2015): Comparison of the 2-step tuberculin skin test and QuantiFERON-TB Gold in-Tube test in the screening of latent tuberculosis infection in cancer patients. Egyptian Journal of Chest Diseases and Tuberculosis. Volume 64, Issue 3, July 2015, Pages 681-688

24. National TB control Program (2008): Ministry of health and population of Egypt, guidelines on management of tuberculosis for non-chest physicians.

25. Ozdemir D, Annakkaya AN, Tarhan G, Sencan I, Cesur S, Balbay O and Guclu E. (2007):Comparison of the tuberculin skin test and the quantiferon test for latent Mycobacterium tuberculosis infections in health care workers in Turkey. Jpn J Infect Dis. May;60(2-3):102-5.

26. Pai M and O'Brien R. (2008): New diagnostics for latent and active tuberculosis: state of the art and future prospects. Semin Respir Crit Care Med. Oct; 29(5):560-8.

27. Pratt A, Nicholl K and Kay L (2007): Use of the Quantiferon TB Gold test as part of a screening programme in patients with RA under consideration for treatment with anti-TNF-alpha agents: The Newcastle (UK) experience. Rheumatology (Oxford) 46: 1035-1036

28. Seyhan EC, Sökücü S, Altin S, Günlüoğlu G, Trablus S, Yilmaz D, Koksalan OK, Issever H.(2010): Comparison of the QuantiFERON-TB Gold In-Tube test with the tuberculin skin test for detecting latent tuberculosis infection in hemodialysis patients. Transpl infect disease 12(2):98-105.

29. Soysal A, Bakir M, Millington KA, Deeks JJ, Efee S, Aslan Y, Dosanjh DP, Lalvani A. (2008): Prognostic value of a T-cell-based, interferon-gamma biomarker in children with tuberculosis contact. Ann Intern Med. Dec 2;149(11):777-87.

30. Taggart EW, Hill HR, Ruegner RG et al. (2004): Evaluation of an in vitro assay for gamma interferon production in response to Mycobacterium tuberculosis infections. ClinDiagn. Lab. Immunol., 11 (6) :1089-1093

31. Tegbaru B, Wolday D, Messele T, Legesse M, Mekonnen Y, Miedema F, van Baarle D. (2006): Tuberculin skin test conversion and reactivity rates among adults with and without human immunodeficiency virus in urban settings in Ethiopia. Clin Vaccine Immunol. Jul;13(7):784-9

32. Triverio PA, Bridevaux PO, Roux-Lombard P, Niksic L, Rochat T, Martin PY, et al. (2009): Interferon-gamma release assays versus tuberculin skin testing for detection of latent tuberculosis in chronic hemodialysis patients. Nephrol Dial Transplant; 24: 1952-6.

33. Wang L, Turner MO, Elwood RK, Schulzer M, FitzGerald JM. (2002): A meta-analysis of the effect of BacillusCalmette-Guerin vaccination on tuberculin skin test measurements. Thorax; 57: 804-9.

34. Winthrop KL, Nyendak M, Calvet H, Oh P, Lo M, Swarbrick G, et al. (2008): Interferon-gamma release assays for diagnosing mycobacterium tuberculosis infection in renal dialysis patients. Clin J Am Soc Nephrol; 3: $1357-63$.

35. World Health Organization (2004): Global tuberculosis control: surveillance, planning, financing. WHO Report. http://www.who.int/tb

36. World Health Organization (2009): Global tuberculosis control: surveillance, planning, financing. Geneva: World Health Organization. p. 1-303. 